\title{
Effect of iron supplementation on mild to moderate anaemia in pulmonary tuberculosis
}

\author{
Uma Devi, C. Mohan Rao, Vinod K. Srivastava, Pramod K. Rath and Bhabani S. Das* \\ Departments of Chest and Tubercular Diseases, Pathology, and Biochemistry, Ispat General Hospital, \\ Rourkela - 769 005, Orissa, India
}

(Received 19 April 2002 - Revised 23 December 2002 - Accepted 10 May 2003)

\begin{abstract}
Anaemia is a common complication of pulmonary tuberculosis. The precise mechanism of anaemia in pulmonary tuberculosis is not clearly known, but anaemia of inflammation as well as of Fe deficiency has been implicated. Both are common in developing countries. It is extremely difficult to distinguish anaemia of Fe deficiency from anaemia of inflammation with the haematological indices used routinely. Therefore, Fe preparations are usually prescribed for all anaemic patients irrespective of the aetiology. This approach has been questioned. The present study aimed to assess the effect of Fe supplementation on anaemic patients with pulmonary tuberculosis. Adult male patients 15-60 years of age with pulmonary tuberculosis and a blood haemoglobin concentration $80-110 \mathrm{~g} / \mathrm{l}$ were included in the study; healthy adult males matched for age and socio-economic status were taken as controls. Blood haemoglobin concentration, total erythrocyte count (TEC), packed cell volume (PCV), mean corpuscular volume (MCV), mean corpuscular haemoglobin and serum Fe, total Fe-binding capacity and ferritin were estimated before treatment and 1,2 and 6 months after treatment. The patients were divided randomly into three groups and during the initial 2 months of treatment were provided with one of three supplementary regimens consisting of placebo, $\mathrm{Fe}$ alone or $\mathrm{Fe}$ with other haematinics. Significant improvements in haematological indices and Fe status were noticed in all three groups. Blood haemoglobin concentration, MCV and PCV were significantly higher at 1 month in both Fe-supplemented groups than the placebo group. This difference, however, disappeared at 2 and 6 months with similar values in all three groups. The increase of other haematological indices was similar in all groups. Serum Fe and Fe saturation of transferrin were significantly higher in both Fe-supplemented groups than the placebo group up to 2 months; this effect, however, disappeared at 6 months. There was a consistent increase in TEC and decrease in ferritin values up to 6 months in all groups. Radiological and clinical improvement was similar in all three groups. These observations suggest that Fe supplementation in mild to moderate anaemia associated with pulmonary tuberculosis accelerated the normal resumption of haematopoiesis in the initial phases by increasing Fe saturation of transferrin. However, consistent improvement of haematological status was dependent only on the improvement of the disease process.
\end{abstract}

Anaemia: Iron: Pulmonary tuberculosis

Anaemia is a common complication of pulmonary tuberculosis, the reported prevalence ranging from 16 to $76 \%$ in different studies (Goldenberg, 1996). A lower blood haemoglobin concentration was found in children with tuberculosis than in non-tubercular children (Wessels et al. 1999). The precise mechanism of anaemia in pulmonary tuberculosis is not known. Several hypotheses, including blood loss because of haemoptysis (blood in the sputum), bone marrow involvement with tubercular granuloma in disseminated tuberculosis (Lombard \& Mansvelt, 1993), nutritional deficiency caused by fever and loss of appetite etc., have been proposed. Anaemia in pulmonary tuberculosis may also occur as a consequence of chronic inflammation, and without apparent loss of blood or bone marrow suppression (Baynes et al. 1986a). Blunted response of erythropoietin due to release of tumour necrosis factor $\alpha$ or other cytokines have been observed (Ebrahim et al. 1995). Tuberculosis is a chronic infectious disease, so anaemia of inflammation may contribute significantly.

Hypoferraemia induced by the shift of Fe from a transferrin-bound available state to a ferritin-incorporated storage state is considered to be a major factor in the pathogenesis of anaemia of chronic inflammation. The condition may have evolved as a cytokine-mediated defence against microbial pathogens, effectively withholding $\mathrm{Fe}$ from microbes, which incidentally also deprives erythroid precursors of their Fe supply (Bullen et al. 1978; Weinberg, 1978; Jurado, 1997). Therefore, Fe-deficiency status may be a protective response against invading microbes: mild anaemia may be a small price to pay for the attenuation of infection. The only effective treatment for anaemia

\footnotetext{
Abbreviations: $\mathrm{MCH}$, mean corpuscular haemoglobin; MCV, mean corpuscular volume; PCV, packed cell volume; TEC, total erythrocyte count; TIBC, total Fe-binding capacity.

* Corresponding author: Dr B. S. Das, fax +91661 2642703/+91661 2510085, email bsdas@hotmail.com
} 
of chronic inflammation is correction of the underlying disorder (Andrews, 1999).

The widespread presence of Fe-deficiency anaemia has been reported from many developing countries (Hercberg et al. 1987; DeMaeyer, 1989; Taylor et al. 1993). In fact, anaemia has become synonymous with Fe deficiency and Fe supplementation is the usual practice in therapy for all anaemic patients in most developing countries irrespective of the aetiology. However, anaemia of inflammation is considered a major contributor to anaemia observed in developing countries (Abshire, 1996) and anaemia of inflammation may even be associated with asymptomatic and sub-clinical infections (Das et al. 1997; van den Broek \& Letsky, 2000). While Fe supplementation is the only therapy for Fe-deficiency anaemia, its efficacy in anaemia of inflammation is questionable. Several studies have indicated deleterious effects associated with Fe supplementation in tubercular infection (Dhople et al. 1996; Gomes et al. 1999; Lounis et al. 2001).

Pulmonary tuberculosis is a chronic infective disease occurring predominantly in socio-economically deprived populations. Therefore, both anaemia of inflammation as well as Fe deficiency may coexist in pulmonary tuberculosis. Both pulmonary tuberculosis and anaemia are widely prevalent in and around Rourkela, Orissa, India. We have conducted a prospective double-blind placebo-controlled study to assess the effect of Fe therapy and supplementation with other haematinics in mild to moderately anaemic pulmonary tuberculosis patients.

\section{Materials and methods}

\section{Study site}

Ispat General Hospital, Rourkela, in Sundergarh district of Orissa State, India, where the study was conducted, is a well-equipped modern hospital with 700 beds. It provides free health-care facilities to the employees of the local Steel Plant and Fertiliser Plant and their family members. The Department of Tuberculosis and Chest Diseases in the hospital has seventy-five beds, two-thirds of which are earmarked for pulmonary tuberculosis patients. The department is managed by qualified chest physicians, with the support of medical officers and paramedical staff. Pulmonary tuberculosis patients are usually hospitalised for a minimum period of 2 months to provide an intensive phase of chemotherapy, and those showing uneventful recovery are advised to continue a maintenance phase of chemotherapy for another 4 months as outpatients. During their hospital stay, all tuberculosis patients are routinely provided with protein-rich $(120-130 \mathrm{~g} / \mathrm{d})$ nutritious diets free of charge from the hospital kitchen. After discharge from the hospital, they go back to their usual diet, which consists mainly of rice or wheat-based preparations, cooked vegetables and grams, and occasional non-vegetarian preparations.

\section{Patients}

Adult male pulmonary tuberculosis patients, 15-60 years of age, consecutively admitted to the chest department from
August 1998 to March 2000 and with a blood haemoglobin concentration of 80-110 g/l, were considered for the present study. Only those patients who were entitled to free medical care and residing in the Steel Township were included for the convenience of the follow-up. Female patients were excluded from the present study to avoid the interference of menstruation, pregnancy and childbirth. Children $(<15$ years of age) and the elderly ( $>60$ years of age) were excluded to avoid the altered Fe metabolism. Critically ill patients and patients with extensive or disseminated tuberculosis were excluded because of the uncertainty of the course of the disease; patients with massive haemoptysis and severe anaemia were excluded because of their requirement for blood transfusion and Fe supplementation. Those with associated chronic diseases, such as chronic renal failure, chronic liver disease, haemoglobinopathy, neoplastic disease and collagen vascular disease, and those who could not complete the initial 2 months of hospitalisation or who expired before the completion of the supplementation therapy were also excluded from the study. Malaria is a common cause of anaemia in Sundergarh district, but none of our patients had malaria parasites in their peripheral blood. Malaria transmission in Rourkela Steel Township is very low, because of the excellent urban infrastructure and effective malaria control programmes.

Fifty healthy adult males, matched for age and socioeconomic status, were taken as controls. The average age of the control group was 40.8 (SD 16.8) years. They were selected from those attending the hospital for a preemployment check-up or from the employees and their dependants attending the hospital for a routine medical check-up. Socio-economic matching was done based on income per month (by seeing the payslip of the employee) and the community they belonged to. All the employees and their dependants are entitled to subsidised housing in the township and free primary education and health-care facilities. Blood samples were collected from them for assessment of haematological and Fe status.

The study was approved by the hospital ethical committee of Ispat General Hospital. Only those who consented orally to participate after explanation of the objectives and protocol were included in the study.

\section{Methods}

Diagnosis of clinically suspected cases of pulmonary tuberculosis was confirmed either bacteriologically by detection of acid-fast bacilli in the sputum and/or radiological examination. Radiological classification of chest lesions was made following the guidelines of the National Tuberculosis Association of the USA (1961). All the chest radiological assessments were done by one of the chest physicians (C. M. R.). Briefly, the lesions were classified as follows: (1) minimal lesion (slight to moderate density involving small part of one or both lungs, the total extent of which should not exceed the volume of one-third of the lung on one side and that do not contain demonstrable cavities); (2) moderately advanced lesions (the lesions may be present in one or both lungs: (i) disseminated lesions of slight to moderate density, total involvement limited to volume of one lung; (ii) dense and confluent lesions that 
are limited to one-third of the volume of one lung; (iii) total diameter of cavities if present must be $<40 \mathrm{~mm}$ ); (3) far advanced lesions (lesions more extensive than moderately advanced lesions).

Pre-treatment assessment of BMI, haematological investigations such as blood haemoglobin and erythrocyte sedimentation rate, biochemical tests for assessment of glycaemic status and tests for liver and renal functions were done in all patients to exclude diabetes mellitus, chronic renal failure and hepatic dysfunction. These investigations were repeated in the first, second and sixth month in all patients. Sputum examination was repeated every 2 weeks until three consecutive samples were reported to be negative; chest X-ray was repeated at the end of the intensive ( 2 months) and maintenance (6 months) phases. This protocol of investigations is practised routinely in all patients of pulmonary tuberculosis admitted to the chest ward.

Blood haemoglobin concentration, total erythrocyte count (TEC), packed cell volume (PCV) and mean corpuscular volume (MCV) were estimated in a blood cell counter (Coulter T-660; Coulter Electronics, Luton, Beds., UK) and mean corpuscular haemoglobin $(\mathrm{MCH})$ was calculated from the haemoglobin and TEC. Erythrocyte sedimentation rate was estimated by the Western Green method. Plasma glucose, serum urea, creatinine, bilirubin, alanine aminotransferase, alkaline phosphatase, total protein, albumin, $\mathrm{Fe}$ and total Fe-binding capacity (TIBC) were estimated on an Olympus AU-400 Autoanalyser (Olympus, Tokyo, Japan) using commercial test kits from Randox Laboratories (Crumlin, Antrim, UK). The $\mathrm{CV}$ for $\mathrm{Fe}$ and TIBC using Randox Assayed Multisera Normal containing $23.8 \mu \mathrm{mol}$ Fe and $36.6 \mu \mathrm{mol}$ TIBC/l were 2.8 and $4.7 \%$ respectively ( $n$ 20). Ferritin was estimated by an ELISA two-step sandwich assay using Boehringer Mannheim test kits (Boehringer Mannheim, Mannheim, Germany). The CV for ferritin were 4.8 and $4.3 \%$ at ferritin concentration of 28 and $467 \mu \mathrm{g} / \mathrm{l}$ respectively. Fe saturation of transferrin was calculated from serum Fe and TIBC and was expressed as \% saturation. For estimation of Fe, TIBC and ferritin, serum was stored at $4^{\circ} \mathrm{C}$ and analysed within $7 \mathrm{~d}$ of collection.

All patients in the study group were commenced on a short course of chemotherapy consisting of four drugs per $d$. The patients were hospitalised for the initial 2 months for administration of intensive supervised treatment, consisting of oral ethambutol $(800 \mathrm{mg})$, isoniazide $(300 \mathrm{mg})$, rifampicin $(450 \mathrm{mg})$ and pyrazinamide $(1500 \mathrm{mg})$ once per d, $30 \mathrm{~min}$ before breakfast. After 2 months of intensive therapy, the patients were discharged from hospital and were advised to continue maintenancephase chemotherapy with rifampicin $(450 \mathrm{mg}$ ) and isoniazide $(300 \mathrm{mg})$ once per $\mathrm{d}$. All the drugs for the intensive and maintenance phases were supplied from the hospital stores free of charge.

Each patient in the study group was supplemented with one capsule twice per d (for details, see later), before breakfast (together with the anti-tubercular drugs) and before dinner. Each capsule consisted of one of the following three preparations: (1) placebo containing $75 \mathrm{mg}$ sucrose; (2) ferrous fumarate containing $75 \mathrm{mg}$ elemental $\mathrm{Fe}$; (3) ferrous fumarate containing $75 \mathrm{mg}$ elemental $\mathrm{Fe}$ with other haematinics (zinc sulfate $25 \mathrm{mg}$, L-histidine hydrochloride $2 \mathrm{mg}$, lysine hydrochloride $12.5 \mathrm{mg}$, glycine hydrochloride $5 \mathrm{mg}$, thiamin $2.5 \mathrm{mg}$, riboflavin $1.5 \mathrm{mg}$, pyridoxine $0.75 \mathrm{mg}$, cyanocobalamin $1.25 \mathrm{mg}$, ascorbic acid $20 \mathrm{mg}$, folic acid $0.25 \mathrm{mg}$ ) (placebo and Fe preparations were provided courtesy of Tablets India Ltd, Chenai, India). The capsules were of similar size and colour. The dosage of $\mathrm{Fe}$ supplementation was kept relatively low, because of the added gastrointestinal adverse reactions to anti-tubercular drugs. Accordingly, the groups were designated as group 1 (placebo group), group 2 (Fe-supplemented group) and group 3 (Fe + other haematinics-supplemented group). The patients, treating physicians and the laboratory were blinded regarding the contents of the capsules. The supplements were distributed sequentially according to the code number of the packets. Symptoms of gastrointestinal upset, such as loss of appetite, nausea, diarrhoea, constipation, flatulence, etc., were recorded from each patient every day during the period of intervention. After discharge from the hospital, supplementation was stopped and the patients were advised to take their usual diet.

\section{Statistical analysis}

Data on age, BMI, haematological indices and TIBC were normally distributed and the significance of difference between groups was assessed by ANOVA followed by a Newman-Keul test to estimate the significance of difference between each pair of groups. Data on erythrocyte sedimentation rate, $\mathrm{Fe}, \mathrm{Fe}$ saturation of transferrin (\%) and ferritin were not normally distributed and non-parametric tests were used for statistical analysis. The significance of difference between the groups was assessed by the one-way repeated measurements ANOVA on ranks followed by Scheffe post hoc pairwise comparison to test the difference between two groups. Differences in the severity of disease measured by chest X-ray were assessed using the $\chi^{2}$ test.

\section{Results}

There were 131 patients fulfilling the recruitment criteria admitted during the period of study and included in the study. Two patients died in the hospital during the initial 2-month intensive phase of chemotherapy, one from the placebo group due to massive haemoptysis and the other from the Fe-supplemented group due to a cerebrovascular accident. The remaining 129 patients, forty-three in each group, completed 2 months of intensive anti-tubercular chemotherapy and Fe-placebo supplementation. Of these, forty from group 1, thirty-eight from group 2 and thirtynine from group 3 were available for follow-up until completion of the 6-month short course of chemotherapy. Improvement in clinical profile, weight gain and radiological clearance of the lung shadow was noticed in all patients at the end of 6 months. Minor adverse drug reactions in the form of decreased appetite and flatulence were noticed in four patients in the Fe-supplemented group, in five in the placebo group and in a further five in the $\mathrm{Fe}+$ other haematinics-supplemented group. The gastrointestinal upsets 
were very mild and did not warrant any intervention or discontinuation of therapy. Moreover, anti-tubercular drugs are also known to cause gastrointestinal upset; therefore, it was not possible to ascertain whether an observed gastrointestinal upset was due to $\mathrm{Fe}$ supplementation or antitubercular drugs. All the 129 patients had uneventful recoveries.

Pre-treatment (baseline) comparison of age, BMI, erythrocyte sedimentation rate and radiological severity of the disease did not reveal significant difference between the groups (Table 1). The age of the control subjects was similar to that of the patient groups. The BMI increased consistently from the baseline in all three groups, but a significant difference $(P<0.01)$ from the pre-treatment value was noticed only at 6 months. The BMI of the groups at 1, 2 and 6 months did not differ significantly. The erythrocyte sedimentation rate decreased significantly and sequentially from pre-treatment to the values at 1,2 and 6 months $(P<0 \cdot 001)$, but the decrease was similar in all groups. There was consistent radiological improvement from baseline to 2 and 6 months as indicated by a decrease in the number of patients with an advanced lesion and an increase in the number of patients with a normal chest radiograph $(P<0.01)$. However, the improvement was similar in all three groups (Table 1).

Baseline haematological indices such as blood haemoglobin, TEC, PCV and Fe nutrition indices such as serum $\mathrm{Fe}, \mathrm{Fe}$ saturation of transferrin and TIBC were lower in all three supplemented groups than in healthy controls $(P<0 \cdot 01)$ as expected, since we selected only anaemic patients. However, MCV and MCH did not differ between the patients and the controls, and serum ferritin was significantly higher in patient groups than the controls $(P<0 \cdot 01)$. Of 129 patients who completed 2 months of intensive

Table 1. Indicators of disease status in the placebo group (group 1), iron-supplemented group (group 2 ) and iron + other haematinicssupplemented group (group 3) at baseline and at 1 and 2 months, and 6 months of anti-tubercular therapy§ (Mean values and standard deviations)

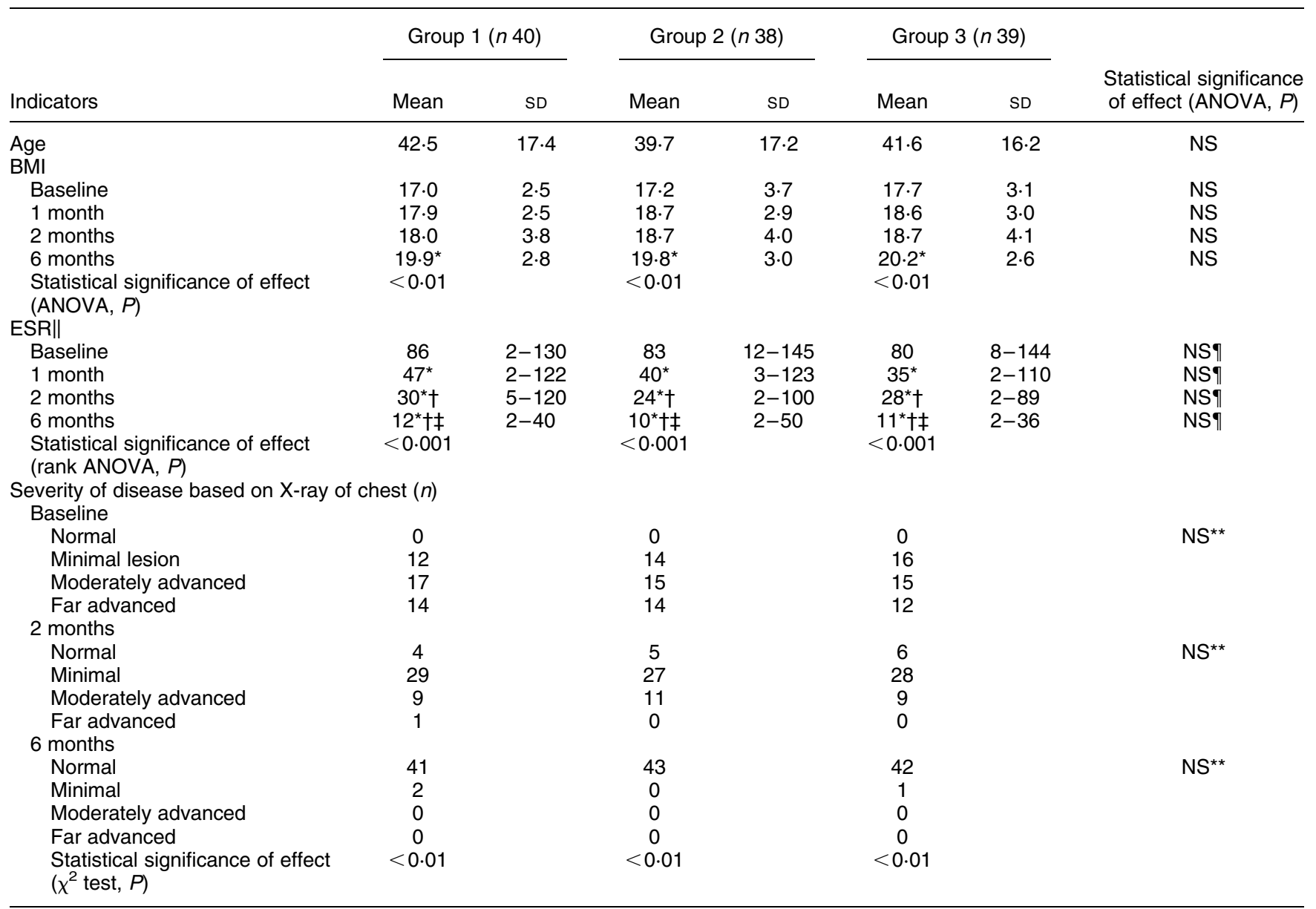

ESR, erythrocyte sedimentation rate.

Mean or median values were significantly different from those at baseline: ${ }^{*} P<0.05$.

Median values were significantly different from those at 1 month: $† P<0.05$.

Median values were significantly different from those at 2 months: $\ddagger P<0.05$

$\S$ For details of subjects and procedures, see p. 542.

$\|$ Median values and ranges.

I Rank ANOVA.

${ }^{\star \star} \chi^{2}$ test. 
Table 2. Haematological indices in the placebo group (group 1), iron-supplemented group (group 2) and iron + other haematinicssupplemented group (group 3) at baseline and at 1 and 2 months after supplementation, and at 6 months of anti-tubercular therapyll

(Mean values and standard deviations)

\begin{tabular}{|c|c|c|c|c|c|c|c|}
\hline \multirow[b]{2}{*}{ Haematological indices } & \multicolumn{2}{|c|}{ Group 1 (n 40) } & \multicolumn{2}{|c|}{ Group 2 (n 38) } & \multicolumn{2}{|c|}{ Group 3 (n 39) } & \multirow{2}{*}{$\begin{array}{l}\text { Statistical significance } \\
\text { of effect (ANOVA, } P \text { ) }\end{array}$} \\
\hline & Mean & SD & Mean & SD & Mean & SD & \\
\hline \multicolumn{8}{|l|}{ Haemoglobin ( $\mathrm{g} / \mathrm{l}$ blood) } \\
\hline Baseline & $95 \cdot 2$ & 11.9 & $95 \cdot 8$ & $10 \cdot 1$ & $93 \cdot 8$ & $11 \cdot 8$ & NS \\
\hline 1 month & $101 \cdot 3$ & $13 \cdot 9$ & $111 \cdot 8^{\star} \S$ & 14.4 & $109 \cdot 9^{\star} \S$ & $13 \cdot 5$ & $<0.01$ \\
\hline 2 months & $110 \cdot 3^{*} \dagger$ & $15 \cdot 7$ & $116 \cdot 0^{*}$ & $13 \cdot 6$ & $116 \cdot 1^{*}$ & $15 \cdot 5$ & NS \\
\hline Statistical significance of effect (ANOVA, $P$ ) & $<0.001$ & & $<0.001$ & & $<0.001$ & & \\
\hline \multicolumn{8}{|l|}{ TEC (cells/nl blood) } \\
\hline Baseline & $3 \cdot 84$ & 0.48 & 3.83 & 0.43 & $3 \cdot 77$ & 0.63 & NS \\
\hline 1 month & $4 \cdot 04$ & 0.49 & $4 \cdot 11^{*}$ & 0.48 & $4 \cdot 15^{\star}$ & 0.55 & NS \\
\hline 2 months & $4 \cdot 24^{*}$ & 0.50 & $4 \cdot 31^{*}$ & 0.47 & $4 \cdot 34^{*}$ & 0.51 & NS \\
\hline 6 months & $4 \cdot 24^{*}$ & 0.56 & $4 \cdot 35^{\star}$ & 0.34 & $4 \cdot 47^{*}$ & 0.63 & NS \\
\hline Statistical significance of effect (ANOVA, $P$ ) & $<0.002$ & & $<0.001$ & & $<0.001$ & & \\
\hline \multicolumn{8}{|l|}{ PCV (\%) } \\
\hline 1 month & $31 \cdot 0$ & $3 \cdot 8$ & $33 \cdot 4^{\star} \S$ & $4 \cdot 4$ & $34 \cdot 0 * \S$ & 3.9 & $<0.01$ \\
\hline 2 months & $33 \cdot 1^{*}$ & $5 \cdot 3$ & $34 \cdot 3^{*}$ & $4 \cdot 4$ & $34 \cdot 8^{*}$ & $4 \cdot 0$ & NS \\
\hline 6 months & $34 \cdot 3^{*} \dagger$ & 5.5 & $36 \cdot 3^{*} \dagger$ & 3.9 & $37 \cdot 2^{*} \dagger \ddagger$ & $4 \cdot 9$ & NS \\
\hline Statistical significance of effect (ANOVA, $P$ ) & $<0.001$ & & $<0.001$ & & $<0.001$ & & \\
\hline \multicolumn{8}{|l|}{ MCV (fl/erythrocyte) } \\
\hline Baseline & $76 \cdot 9$ & $6 \cdot 2$ & $78 \cdot 4$ & $7 \cdot 7$ & $79 \cdot 5$ & $13 \cdot 5$ & NS \\
\hline 1 month & $76 \cdot 9$ & $7 \cdot \overline{6}$ & $81.6 \S$ & $9 \cdot 6$ & $82 \cdot 6 \S$ & $10 \cdot 8$ & $<0.05$ \\
\hline 2 months & $78 \cdot 3$ & $10 \cdot 2$ & $80 \cdot 1$ & $10 \cdot 1$ & $81 \cdot 0$ & $10 \cdot 3$ & NS \\
\hline 6 months & $81 \cdot 0$ & $8 \cdot 5$ & $83 \cdot 7$ & $9 \cdot 3$ & $84 \cdot 0$ & $11 \cdot 7$ & NS \\
\hline Statistical significance of effect (ANOVA, $P$ ) & NS & & NS & & NS & & \\
\hline \multicolumn{8}{|l|}{$\mathrm{MCH}$ (pg/erythrocyte) } \\
\hline Baseline & $24 \cdot 9$ & $2 \cdot 6$ & $25 \cdot 3$ & $3 \cdot 0$ & $25 \cdot 3$ & $4 \cdot 1$ & NS \\
\hline 1 month & $25 \cdot 2$ & $2 \cdot 9$ & $27 \cdot 4$ & $3 \cdot 7$ & $26 \cdot 8$ & 3.9 & NS \\
\hline 2 months & $26 \cdot 2$ & $3 \cdot 5$ & $27 \cdot 2$ & $3 \cdot 3$ & $27 \cdot 0$ & 3.9 & NS \\
\hline 6 months & $27 \cdot 4^{*} \dagger$ & 3.7 & $27 \cdot 6^{*}$ & $3 \cdot 3$ & $27 \cdot 2$ & $4 \cdot 0$ & NS \\
\hline Statistical significance of effect (ANOVA, $P$ ) & $<0.02$ & & $<0.02$ & & NS & & \\
\hline
\end{tabular}

TEC, total erythrocyte count; PCV, packed cell volume; MCV, mean corpuscular volume; $\mathrm{MCH}$, mean corpuscular haemoglobin.

Mean values were significantly different from those at baseline: ${ }^{\star} P<0.05$.

Mean values were significantly different from those at 1 month: $\dagger P<0.05$.

Mean value was significantly different from that at 2 months: $\ddagger P<0.05$.

Mean values were significantly different from those of the placebo group: $\S P<0.05$

|l For details of subjects and procedures, see p. 542.

chemotherapy, seventy-eight had microcytosis, ninety-two had hypochromia and sixty-nine had both microcytosis and hypochromia.

Baseline haematological indices did not differ significantly between the three patient groups (Table 2). During follow-up, haematological indices improved steadily and sequentially in all groups, although the magnitude of increase was different. The increase in blood haemoglobin concentration from baseline to 1 month was significantly higher in both Fe-supplemented groups (groups 2 and 3); however, in the placebo group, although there was an increase, it was not statistically significant. Thus, blood haemoglobin concentration at 1 month was higher in both the Fe-supplemented groups than in the placebo group $(P<0.01)$. However, the increase in blood haemoglobin from the first to the second month was not statistically significant in groups 2 and 3, but in group 1 the increase was significant from values at baseline and at 1 month. The values at 6 months were significantly higher than those at baseline and 1 month in groups 1 and 3 , and from baseline values only in group 2 (Table 2). Similarly, TEC and PCV increased significantly from the baseline at 1 month in groups 2 and 3 , but not in group 1 , which showed significant increase from baseline only at 2 months. At 6 months, while blood TEC showed significant increase only from baseline values in all three groups, blood PCV was significantly higher than values at baseline and 1 month in groups 1 and 2, and from all the three previous values in group 3 . The increases in $\mathrm{MCV}$ and $\mathrm{MCH}$ were not statistically significant during the period of follow-up, except for $\mathrm{MCH}$ values at 6 months in groups 1 and 2 (Table 2). Overall, there was a consistent increase in haematological indices in all groups and the values at different periods of follow-up were not statistically different except for blood haemoglobin, PCV and MCV, which were higher at 1 month in groups 2 and 3 in comparison with group 1 .

$\mathrm{Fe}$ nutrition status of the groups at baseline, 1, 2 and 6 months after treatment is shown in Table 3. The pretreatment serum $\mathrm{Fe}$ values were very low in all three groups, and these increased sequentially and steadily $(P<0.001)$. There was a significant increase from the baseline values at 1 month in all three groups, and from 1 to 2 months in groups 1 and 3 , but not in group 2 . 


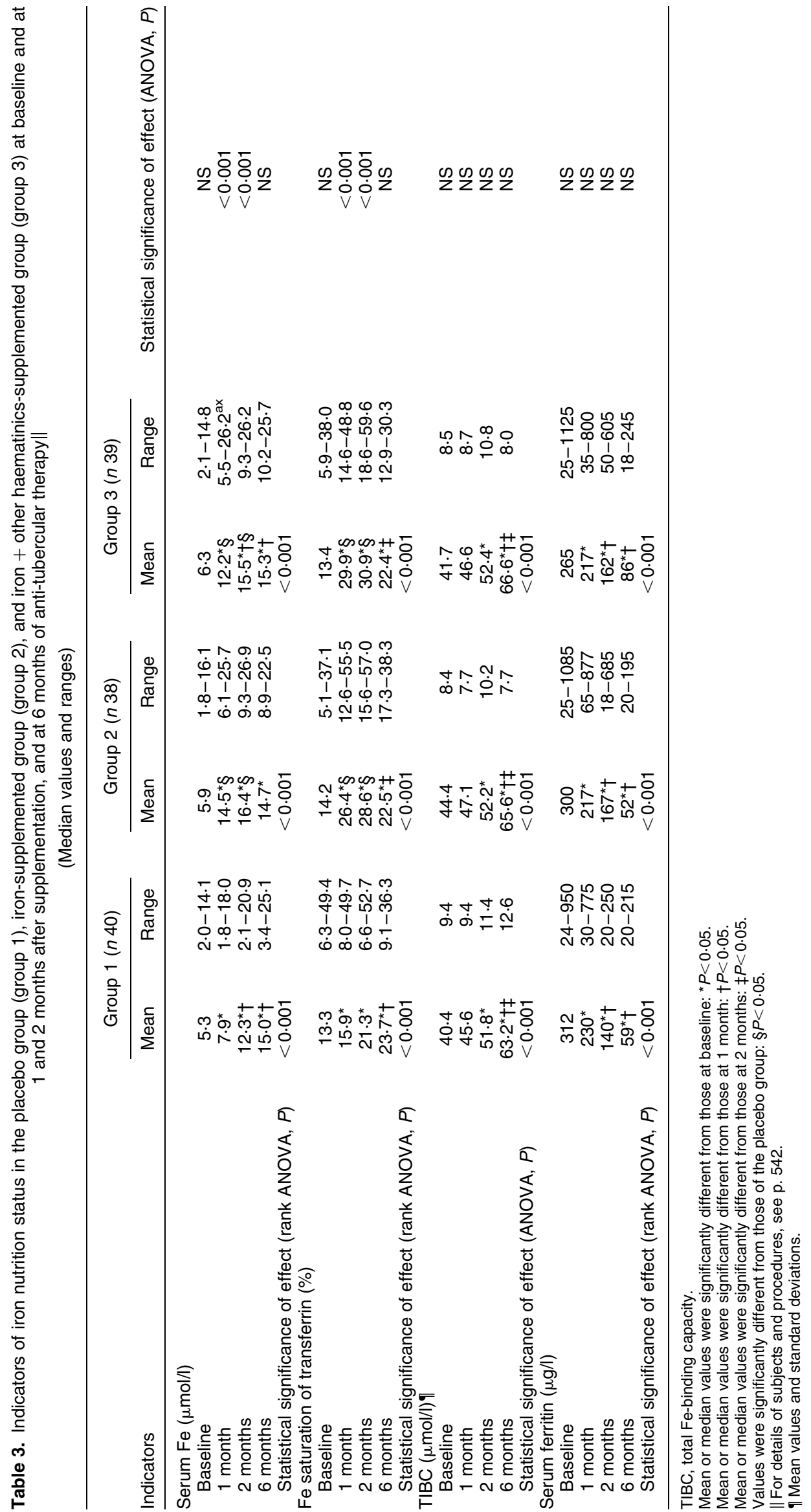


The increase was significantly higher in both the Fe-supplemented groups than in the placebo group, and this is reflected in the significantly higher serum Fe concentrations at 1 and 2 months in Fe-supplemented groups than the placebo group $(P<001)$. The increase continued up to 6 months in the placebo group but not in the Fe-supplemented groups. The difference in the serum Fe concentration noticed between the $\mathrm{Fe}$-supplemented groups and the placebo group at 1 and 2 months disappeared at 6 months (Table 3).

Fe saturation of transferrin increased simultaneously with the increase in serum $\mathrm{Fe}$ concentrations, and a statistically significant increase from the baseline value was noticed at 1 and 2 months in all groups. However, the increase continued up to 6 months only in the placebo group, while the $\mathrm{Fe}$-supplemented groups had significantly lower Fe saturation of transferrin at 6 months compared with values at 2 months. Similar to serum Fe, Fe saturation of transferrin was significantly higher in Fe supplemented groups than the placebo group at 1 and 2 months $(P<0.001)$, but this effect disappeared at 6 months (Table 3).

Pre-treatment TIBC values were low in all groups, and these showed a steady and consistent increase during the period of study. The increase was almost similar in all the groups and unlike serum $\mathrm{Fe}$ and $\mathrm{Fe}$ saturation of transferrin, TIBC values at 1,2 and 6 months were not significantly different between the supplemented and placebo groups (Table 3).

Baseline serum ferritin concentration was high in all three groups. This decreased sequentially up to 6 months $(P<0.001)$. Although the decrease continued up to 6 months, it was statistically significant only up to 2 months. The decrease was almost similar in all the three groups, as indicated by an insignificant difference between the different groups both at baseline and after supplementation. However, high ferritin values persisted even after 6 months of effective anti-tubercular therapy (Table 3).

After 2 months of supplementation, blood haemoglobin concentration and PCV were still significantly lower than that of matched controls, but TEC, MCV and $\mathrm{MCH}$ were not significantly different. Among the Fe nutrition indices, TIBC was significantly low and ferritin was significantly high in all three supplemented groups in comparison with control values. Serum Fe was higher in group 2 than in the placebo group and in group 3 than in the control as well as placebo groups. Fe saturation of transferrin was significantly higher in groups 2 and 3 than both control and placebo group (Table 4). These differences disappeared at 6 months except for serum ferritin, which remained persistently high in all the three groups in comparison with control values (Table 4).

\section{Discussion}

Both Fe deficiency and anaemia of chronic inflammation may coexist in patients with pulmonary tuberculosis, especially in developing countries. Anaemia of chronic inflammation has several features in common with Fe-deficiency anaemia, thus confusing the aetiological diagnosis. Raised erythrocyte volume distribution width, a hallmark of Fe-deficiency anaemia, is also observed in anaemic tuberculosis patients (Baynes et al. 1986b). Other characteristic laboratory findings of Fe-deficiency anaemia, such as low $\mathrm{Fe}$ and transferrin saturation in blood and hypochromic and microcytic erythrocytes, are all seen in anaemia of inflammation (Das et al. 1997, 1999). It is, therefore, difficult to establish the exact mechanism of associated anaemia in pulmonary tuberculosis patients with the routine investigations undertaken for diagnosis of anaemia

In the present study, low MCV and $\mathrm{MCH}$ values were observed in a significant proportion of patients. Although it is quite reasonable to think that such widespread microcytosis and hypochromia would not be expected in anaemia of inflammation alone, there is a growing body of opinion that $\mathrm{Fe}$ deficiency is a far less important cause of anaemia in developing countries than previously believed. Both symptomatic and asymptomatic infections are considered to be major contributory factors to anaemia seen in developing countries (Das et al. 1997). A recent study conducted to analyse the mechanism of anaemia in pregnant women found that more than half of anaemic women had markers of inflammation (Nynke et al. 2000). The mechanisms causing low serum Fe and Fe saturation of transferrin seen in our present patients could be due to either Fe deficiency or anaemia of inflammation (Jurado, 1997), or both.

Transferrin and TIBC values are usually high in Fe-deficiency anaemia and low in anaemia of inflammation (Fleck \& Myers, 1985; Punnonen et al. 1994). The low TIBC concentrations seen in our present patients might have been the result of an acute-phase response. Pretreatment serum ferritin concentration was high in all three groups. Ferritin concentration in blood is considered to be a specific indicator of body Fe stores (Lipschitz et al. 1974); however, the concentrations can rise following an inflammatory response, irrespective of $\mathrm{Fe}$ status (Dallman et al. 1981; Harju et al. 1984; Henderson, 1984; Fitzsimons \& Govostis, 1986; Taylor et al. 1993). Recent reports indicate that ferritin synthesis is stimulated in pulmonary tuberculosis as a consequence of the inflammatory process (Wessels et al. 1999). Raised ferritin and low TIBC values in the face of extensive microcytosis and hypochromia seen in our present patients point more towards the possibility of anaemia of inflammation, although association of Fe-deficiency anaemia cannot be completely ruled out.

The result of $\mathrm{Fe}$ supplementation in the present study was a persistent and sequential increase in blood haemoglobin, TEC and PCV in all groups. The increase in blood haemoglobin, PCV and MCV was significantly higher in the $\mathrm{Fe}$-supplemented groups than in the placebo group at 1 month. The differential increase may indicate that there could be some degree of associated $\mathrm{Fe}$ deficiency, which improved more rapidly on $\mathrm{Fe}$ supplementation, or that Fe supplementation accelerated the normal resumption of haematopoiesis in the initial phases by increasing $\mathrm{Fe}$ saturation of transferrin. The difference noticed between the placebo and Fe-supplemented groups at 1 month disappeared at 2 months despite continuation of $\mathrm{Fe}$ supplementation up to 2 months (Table 2) 
U. Devi et al.

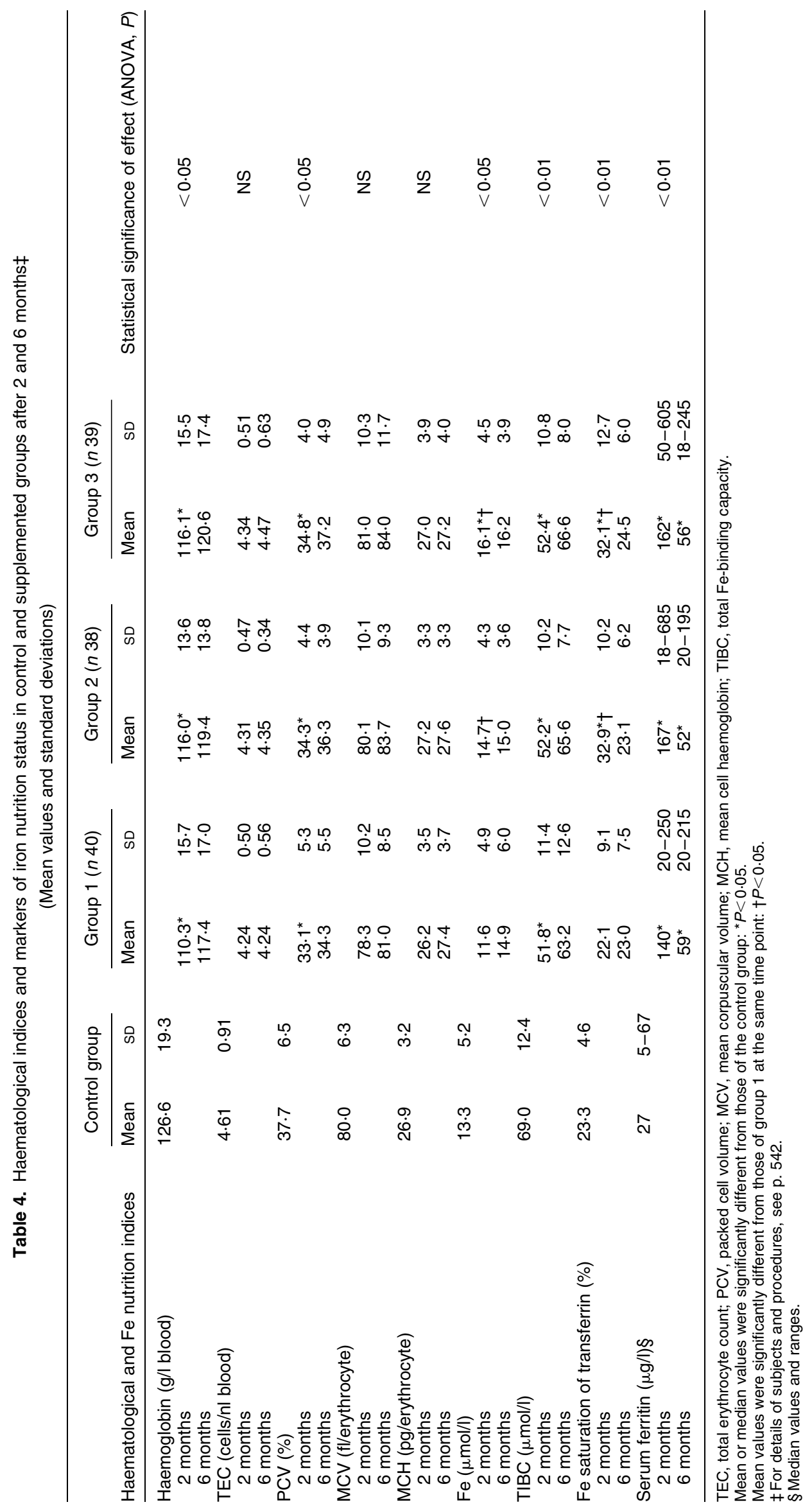


and persistence of anaemia (Table 4). After initial faster improvement in haematological indices in the Fe-supplemented groups, the increase slowed, possibly because inflammation contributed significantly towards anaemia and further improvement was dependent more on the correction of the inflammatory process and not $\mathrm{Fe}$ supplementation.

It is believed that in anaemia of inflammation, Fe deposited in the monocyte-macrophage system is not mobilised enough for adequate transferrin saturation. Macrophage $\mathrm{Fe}$ becomes available for erythropoiesis through two mobilisation pathways: a rapid pathway associated with immediate return of $\mathrm{Fe}$ retrieved from senescent erythrocytes and a slower pathway consisting of $\mathrm{Fe}$ mobilised from storage sites. In the anaemia of chronic disorders, the slower pathway may predominate (Robert \& Means, 1999). Non-availability of $\mathrm{Fe}$ for erythropoiesis is partly responsible for inadequate marrow response, leading to microcytosis and hypochromia (Abshire, 1996). However, ferrokinetic studies have yielded conflicting results: while erythroid-Fe turnover correlated with serum Fe level in one study (Douglas \& Adamson, 1975), indicating that the marrow proliferation was limited by non-availability of $\mathrm{Fe}$, another found no correlation (Cavill \& Bentley, 1982). Based on the presumption that hypoferraemia was the main cause of anaemia of inflammation, intravenous Fe supplementation was tested (Bentley \& Williams, 1982). Although some improvement was noticed, acceptance of the therapeutic regimen was poor, because the adverse reactions to the intravenous route of supplementation far exceeded the low likelihood of benefit. It has been reported that oral Fe-replacement therapy in the anaemia of chronic disease gives benefit only if concurrent $\mathrm{Fe}$ deficiency exists and corrects that component of anaemia caused by Fe deficiency (Baer et al. 1990).

There was a significant improvement in serum Fe, TIBC and $\mathrm{Fe}$ saturation of transferrin in all three groups. It appears that TIBC returned towards normal with the decrease in the acute-phase response during the recovery from the disease process. The increase in TIBC was similar in the Fe-supplemented and placebo groups. Thus, increase in TIBC values appeared to be dependent on recovery from the inflammatory process and independent of $\mathrm{Fe}$ supplementation. Serum $\mathrm{Fe}$ and Fe saturation of transferrin were higher in the supplemented groups than in the placebo group during the period of supplementation $(P<0.001)$. After withdrawal of Fe supplementation, Fe saturation of transferrin decreased in the supplemented groups and values at 6 months were lower than those at 2 months, while in the placebo group there was an increase at 6 months from the 2 months value (Table 3).

We next wanted to examine if Fe supplementation in pulmonary tuberculosis is harmful. Fe functions as a coenzyme for several important enzymes, particularly those involved in electron transport. Hence, it is essential for survival of most living organisms. During infection, the human host withholds Fe as a defence mechanism against micro-organisms, depriving them of this critical nutrient (Bullen et al. 1978; Weinberg, 1978). Fe supplementation resulted in increased bacterial load in liver, spleen and lungs in mice inoculated with Mycobacterium avium (Dhople et al. 1996). Fe loading also significantly enhanced growth of a virulent strain of $M$. tuberculosis in the spleen and lungs of female Balb/C mice. It was concluded that an excess of $\mathrm{Fe}$ might enhance the growth of M. tuberculosis and worsen the outcome of human tuberculosis (Lounis et al. 2001). An Fe-poor diet led to reduced $M$. avium proliferation in mice and Fe-chelating compounds were suggested as useful adjunct therapy (Gomes et al. 1999). However, we did not notice any difference in the clinical and radiological improvement between the Fe-supplemented groups and placebo group (Table 1). Gastrointestinal upset was noticed in four patients in the Fe-supplemented group, in five in the $\mathrm{Fe}+$ other haematinics-supplemented group and in five in the placebo group. However, the symptoms were mild and did not warrant discontinuation of supplementation. Like most bacteria, $M$. tuberculosis is able to acquire Fe from the host for its survival. Mycobacteria respond to Fe starvation by inducing the synthesis and secretion of siderophores, which solubilise Fe and allow it to be transported across the cell envelope via specific receptors (Gobin et al. 1995). Simultaneously, all mycobacteria also contain an Fe-dependent regulator, which negatively regulates siderophore production under $\mathrm{Fe}$-sufficient conditions (Dussurget et al. 1996; Rodriguez et al. 1999). Therefore, it is possible that $M$. tuberculosis possesses regulatory mechanisms for $\mathrm{Fe}$ extraction from the host to take care of fluctuations in in vivo Fe content. Thus, Fe supplementation to correct anaemia in pulmonary tuberculosis may not influence growth and multiplication of mycobacteria significantly and hence severity of the disease process.

Pulmonary tuberculosis patients in our hospital are routinely provided with a high-energy, protein-rich diet during their hospital stay; the patients in the present study also received this diet. We have no knowledge of the $\mathrm{Fe}$ content of these diets and their contribution to correction of anaemia. A very large proportion of tuberculosis patients does not have access to such good diets and therefore, results of the present study cannot be extrapolated to all tuberculosis patients. The contribution of a protein-rich nutritious diet in correcting anaemia of tuberculosis, and the influence of $\mathrm{Fe}$ supplementation in correcting anaemia in patients receiving their natural diets, needs to be evaluated.

In conclusion, inflammation appears to be a major contributor to anaemia associated with pulmonary tuberculosis. Fe and other haematinic supplements can initiate an initial improvement in some haematological indices, but ultimate recovery from anaemia occurs only with recovery from the pulmonary tuberculosis.

\section{Acknowledgements}

The authors acknowledge the Director, Medical and Health Services, Ispat General Hospital for permission to conduct the study. We also thank Drs G. Tripathy, S. Rath, R. Rath, G. Behera and B. Biswas, Department of Respiratory Medicine, Ispat General Hospital, Rourkela, for their cooperation. 


\section{References}

Abshire TC (1996) The anaemia of inflammation: a common cause of childhood anaemia. Pediatr Clinics North America 43, 623-638.

Andrews NC (1999) Disorders of iron metabolism. New Eng J Med 341, 1986-1995.

Baynes RD, Flax H, Bothwell TH, et al. (1986a) Haematological and iron related measurements in active pulmonary tuberculosis. Scand J Haematol 36, 280-287.

Baynes RD, Flax H, Bothwell TH, Bezwoda WR, Atkinson P \& Mendelow B (1986b) Red blood cell distribution width in the anaemia secondary to tuberculosis. Am J Clin Pathol 85, 226-229.

Baer AN, Dessypris EN \& Krantz SB (1990) The pathogenesis of anaemia in rheumatoid arthritis, a clinical and laboratory analysis. Semin Arthritis Rheum 14, 209-223.

Bentley DP \& Williams P (1982) Parenteral iron therapy in the anaemia of rheumatoid arthritis. Rheumatol Rehabil 21, 88-92.

Bullen JJ, Rogers HJ \& Griffiths E (1978) Role of iron in bacterial infection. Curr Top Microbiol Immunol 80, 1-35.

Cavill I \& Bentley DP (1982) Erythropoiesis in the anaemia of rheumatoid arthritis. Br J Haematol 50, 583-590.

Dallman PR, Reeves JD, Driggers DA \& Lo EYT (1981) Diagnosis of iron deficiency: the limitations of laboratory tests in predicting response to iron treatment in 1-year-old infants. J Pediatr 98, 376-381.

Das BS, Nanda NK, Rath PK, Satapathy RN \& Das DB (1999) Anaemia in acute, Plasmodium falciparum malaria in children from Orissa state, India. Ann Trop Med Parasitol 93, 109-118.

Das BS, Thurnham DI \& Das DB (1997) Influence of malaria on markers of iron status in children: implications for interpreting iron status in malaria-endemic communities. Br J Nutr 78, $751-760$.

DeMaeyer EM (1989) Preventing and Controlling Iron Deficiency Anaemia through Primary Health Care: A Guide for Health Administrators and Programme Managers. Geneva: WHO.

Dhople AM, Ibanez MA \& Poirier TC (1996) Role of iron in the pathogenesis of Mycobacterium avium infection in mice. Microbiology 87, 77-87.

Douglas SW \& Adamson JW (1975) The anaemia of chronic disorders: Studies of marrow regulation and iron metabolism. Blood 45, 55-65.

Dussurget O, Rodriguez GM \& Smith I (1996) An ideR mutant of Mycobacterium smegmatis has a depressed siderophore production and an altered oxidative stress response. Mol Microbiol 22, 535-544.

Ebrahim O, Folb PI, Robson SC \& Jacobs P (1995) Blunted erythropoietin response to anaemia in tuberculosis. Eur J Haematol 55, 251-254.

Fitzsimons E \& Govostis M (1986) Changes in serum iron and ferritin concentrations associated with surgery. Clin Chem 32, 201.

Fleck A \& Myers MA (1985) Diagnostic and prognostic significance of acute phase proteins. In The Acute Phase Response to Injury and Infection, pp. 249-271 [AH Gordon and A Koj, editors]. Amsterdam: Elsevier Science Publishers.
Gobin J, Moore CH, Reeve JR, Wong DK, Gibson BW \& Horwitz MA (1995) Iron acquisition by Mycobacterium tuberculosis: Isolation and characterization of a family of iron-binding exochelins. Proc Natl Acad Sci USA 92, 5189-5193.

Goldenberg AS (1996) Haematological abnormalities and mycobacterial infections. In Tuberculosis, pp. 645-652 [WN Rome and S Garay, editors]. Boston, MA: Little Brown and Company.

Gomes MS, Dom G, Pedrosa J, Boelaert JR \& Appelberg R (1999) Effect of iron deprivation on Mycobacterium avium growth. Tuber Lung Dis 79, 321-328.

Harju E, Pakarinen A \& Larmi T (1984) A comparison between serum ferritin concentration and the amount of bone marrow stainable iron. Scand J Clin Lab Invest 44, 555-556.

Henderson A (1984) Ferritin levels in patients with microcytic anaemia complicating pulmonary tuberculosis. Tubercle $\mathbf{6 5}$, $185-189$.

Hercberg S, Galan P \& Dupin H (1987) Iron deficiency in Africa. World Review Nut Diet 54, 201-236.

Jurado RI (1997) Iron, infection, and anaemia of inflammation. Clin Infect Dis 25, 888-895.

Lipschitz DA, Cook JD \& Finch CA (1974) A clinical evaluation of serum ferritin as an index of iron status. New Eng J Med 290, 1213-1216

Lombard EH \& Mansvelt EP (1993) Haematological changes associated with milliary tuberculosis of bone marrow. Tuber Lung Dis 74, 131-135.

Lounis N, Truffot-Pernot C, Grosset J, Gordeuk VR \& Boelaert JR (2001) Iron and mycobacterium tuberculosis infection. J Clin Virol 20, 123-126.

National Tuberculosis Association of USA (1961) Diagnostic Standards and Classification of Tuberculosis. New York: National Tuberculosis Association.

Punnonen K, Irjala K \& Rajamaki A (1994) Iron deficiency anaemia is associated with high concentrations of transferrin receptor in serum. Clin Chem 40, 774-776.

Rafael LJ (1997) Iron, infection and anaemia of inflammation. Clin Infect Dis 25, 888-895.

Robert T \& Means JR (1999) The Anaemia of Chronic Disorders. In Wintrobe's Clinical Haematology, pp. 979-1010 [R Lee and J Foerster, editors]. Baltimore, OH: Willams and Wilkins.

Rodriguez GM, Gold B, Gomez M, Dussurget O \& Smith I (1999) Identification and characterization of two divergently transcribed iron regulated genes. Tuber Lung Dis 79, 287-298.

Taylor PG, Martinez-Torres C \& Mendez-Castellano H, et al. (1993) The relationship between iron deficiency and anaemia in Venezuelan children. Am J Clin Nutr 58, 215-218.

van den Broek NR \& Letsky EA (2000) Etiology of anaemia in pregnancy in south Malawi. Am J Clin Nutr 72, Suppl., $47 \mathrm{~s}-56 \mathrm{~s}$.

Weinberg E (1978) Iron and infection. Microbiol Rev 42, 45-66.

Wessels G, Schaaf HS, Beyers N, Gie RP, Nel E \& Donald PR (1999) Haematological abnormalities in children with tuberculosis. J Trop Pediatr 45, 307-310. 